Research Paper

\title{
A Randomized, Open-Label, Safety and Exploratory Efficacy Study of Kanglaite Injection (KLTi) plus Gemcitabine versus Gemcitabine in Patients with Advanced Pancreatic Cancer
}

\author{
Lee S. Schwartzberg1, Francis P. Arena², Bryan J. Bienvenu³, Edward H. Kaplan4, Luis H. Camacho5, Luis T. \\ Campos $^{6}$, J. Paul Waymack ${ }^{7}$, Mary A. Tagliaferri ${ }^{7}$, Michael M. Chen ${ }^{7}$, Dapeng Li ${ }^{7 凶}$
}

1. West Cancer Center, Germantown, TN

2. Arena Oncology Associates, New York, NY

3. Mary Bird Perkins Cancer Center, Baton Rouge, LA

4. Hematology Oncology of the North Shore, IL

5. St. Luke's Cancer Center, Houston, TX

6. Oncology Consultants, Houston, TX

7. KangLaiTe USA, Redwood City, CA

$\square$ Corresponding author: Dr. Lee Schwartzberg, MD, West Cancer Center 7945 Wolf River Blvd, Germantown TN 38138; Tel: (901) 683-0055; E-mail: lschwartzberg@westclinic.com

(c) Ivyspring International Publisher. This is an open access article distributed under the terms of the Creative Commons Attribution (CC BY-NC) license (https://creativecommons.org/licenses/by-nc/4.0/). See http://ivyspring.com/terms for full terms and conditions.

Received: 2016.02.29; Accepted: 2017.03.22; Published: 2017.07.03

\begin{abstract}
Background: This study was designed to assess the safety and preliminary efficacy of KLTi plus gemcitabine in patients with locally advanced or metastatic pancreatic cancer.

Methods: In a randomized, open-label study, patients with locally advanced or metastatic pancreatic cancer were randomized 2:1 to receive KLTi plus gemcitabine or gemcitabine monotherapy. Three sequential cohorts were tested at $30 \mathrm{~g} /$ day, $50 \mathrm{~g} /$ day, and $30 \mathrm{~g} /$ day. Gemcitabine was administered at $1000 \mathrm{mg} / \mathrm{m}^{2}$ on days 1,8 and 15 of each 28 day cycle. KLTi was administered on days $1-5,8-12$, and $15-19$ of each 28 day cycle. Patients received study treatment until disease progression. The primary endpoint was progression-free survival in the ITT population. Safety evaluation was based on patients who received any study treatment. ClinicalTrials.gov identifier NCT00733850.

Results: Eighty-five patients were randomized including 41 (28:13) in Cohort 1, 18 (12:6) in Cohort 2, and 26 (17:9) in Cohort 3. Due to a different dose and/or shift in patient populations in Cohort 2 and 3, efficacy data for the $30 \mathrm{gm}$ dose are presented in this manuscript for Cohort 1 alone, and for the combination of Cohort $1+3$. The $30 \mathrm{gm} \mathrm{KLTi}+$ gemcitabine group had a statistically significant improvement in progression-free survival (PFS) as assessed by blinded independent radiology review in the ITT population, with a median of 112 days, versus 58 days in the gemcitabine group (HR 0.50; 95\% $\mathrm{Cl}: 0.27,0.92), p=0.0240$. The incidence rates of TEAEs, CTCAE Grade 3 or higher TEAEs, and SAEs were similar between the two arms. There were no deaths related to $\mathrm{KLTi}+$ gemcitabine treatment.

Conclusion: Kanglaite Injection ( $30 \mathrm{~g} /$ day) plus a standard regimen of gemcitabine demonstrated encouraging clinical evidence of anti-neoplastic activity and a well-tolerated safety profile.
\end{abstract}

Key words: Kanglaite Injection, pancreatic cancer, coix seed, traditional Chinese medicinal herb.

\section{Introduction}

Pancreatic cancer is the fourth leading cause of cancer-related death in the United States and Europe, with 1 and 5 year survival rates of $28 \%$ and $7 \%$ among patients of all stages (1). Among patients with advanced diseases, the 5-year survival rate has improved minimally over a 30 -year period (2) from 
$3.1 \%$ to $6.9 \%$ while 1 -year survival rates of 17 to $23 \%$ have been reported with gemcitabine. Current first-line treatment options for unresectable locally advanced or metastatic pancreatic cancer include: gemcitabine monotherapy, which for many years has been the standard therapy(3); erlotinib in combination with gemcitabine, approved by the FDA in 2005, though the improvement in median overall survival was only 2 weeks ${ }^{(4,5)}$; FOLFIRINOX (oxaliplatin, irinotecan, fluorouracil, and leucovorin), which demonstrated an increase of 4.3 months in median overall survival in 2010 from a phase 2-3 trial conducted by a French consortium study group, though its toxicity limits its use to highly selective groups of patients( ${ }^{(6)}$; and nab-paclitaxel (Abraxane) in combination with gemcitabine - approved by the FDA in 2013 - with an improvement in median overall survival of 1.8 months $(7,8)$.

Kanglaite Injection is a neutral oil extracted and isolated from coix seed (a member of the grass family; Coix lacryma-jobi), a traditional Chinese medicinal herb used in China for therapeutic purposes for thousands of years. It has been recorded in many ancient Chinese medicinal books and has been compiled in editions of Chinese Pharmacopoeia as a traditional medicine. It was approved in China in 1995 for the treatment of advanced non-small cell lung cancer and advanced hepatocellular carcinoma (9). Additionally, it is used to treat cancer cachexia, cancer-related pain, and other systemic manifestations of the disease. Over 1 million patients in multiple countries have received KLTi over the past 20 years without serious toxicities.

In preclinical studies, KLTi showed anti-tumor activity as a single agent and synergistic activity in combination with gemcitabine in animal models of pancreatic cancer. ${ }^{(10)}$ Preclinical investigation of KLTi revealed a pleotropic biologic effect in malignant cell lines, including enhancement of apoptosis and down regulation of TNG-alpha, NFkB and COX2(11,12). A phase I dose escalation study in sixteen patients with advanced solid tumors refractory to standard therapy, conducted at University of Utah Huntsman Cancer Institute, evaluated $10 \mathrm{~g}, 20 \mathrm{~g}, 30 \mathrm{~g}, 40 \mathrm{~g}$, and $50 \mathrm{~g}$ dose levels and reported KLTi to be safe with few serious adverse events and none attributed to the drug. The maximum tolerated dose was not reached in the study.(13) Preliminary promising results were reported from a Chinese open-label, randomized, multicenter trial comparing gemcitabine monotherapy to KLTi plus gemcitabine as first line treatment in patients with advanced pancreatic carcinoma. (14) This phase $2 \mathrm{~b}$ study was initiated to further investigate the safety and efficacy of KLTi in combination with gemcitabine compared to gemcitabine monotherapy in US patients with advanced pancreatic cancer.

\section{Methods}

\section{Study design}

This phase $2 \mathrm{~b}$, multi-center, randomized, open-label clinical trial was designed to evaluate the safety and efficacy of KLTi plus gemcitabine versus gemcitabine monotherapy, in a 2:1 randomization ratio, in patients with locally advanced or metastatic pancreatic cancer, not amendable to curative surgical resection. Twenty-one US cancer centers participated in the study. All participating sites received Institutional Review Board (IRB) approval for all versions of the protocol and informed consent prior to enrollment of patients. Three sequential cohorts were enrolled. Cohort $1 \& 2$ were planned to evaluate KLTi $30 \mathrm{~g} /$ day \& $50 \mathrm{~g} /$ day doses, respectively, plus standard doses of gemcitabine versus a standard gemcitabine regimen. After Cohort 2 was closed due to enrollment difficulties after FOLFIRINOX became available, a third cohort, Cohort 3, was added to evaluate a potential PFS benefit of Kanglaite Injection $30 \mathrm{~g} /$ day dose, with a goal of an additional 100 patients. Gemcitabine was administered at 1000 $\mathrm{mg} / \mathrm{m}^{2}$ on days 1,8 and 15 for each 28 day cycle. Kanglaite Injection was administered on days 1-5, 8-12, and 15-19 of each 28 day cycle.

Treatment continued until disease progression, or until there was unacceptable toxicity, or until patients withdrew consent. Gemcitabine-only patients could elect to receive KLTi in an open-label extension after study treatment discontinuation from the main study.

\section{Patients}

Inclusion criteria included a life expectancy $\geq 84$ days (12 weeks), ambulatory states and either a Karnofsky performance status (KPS) of $\geq 60$ or Eastern Cooperative Oncology Group (ECOG) performance status of 0,1 or 2, plus histologically (cytological) confirmed pancreatic cancer, either locally invasive or metastatic, and not amenable to potentially curative surgical resection. Patients must not have previously received chemotherapy for metastatic disease and must have measurable or evaluable target lesions, by RECIST 1.1 criteria.

Exclusion criteria included a history of cancer within 5 years other than pancreatic cancer (excluding resected non-melanoma skin carcinoma); active (untreated or still receiving corticosteroids) brain metastases; prior gemcitabine $<12$ months; taking a bile acid sequestrant drug or a fibric acid derivative drug; disturbances of lipid metabolism such as pathologic hyperlipidemia (including congenital hypertriglyceridemia or cholesterolemia), lipoid 
nephrosis, or acute pancreatitis; or uncontrolled Type 1 or 2 diabetes mellitus.

Patients were randomized between August 25, 2008 and February 7, 2014. All patients provided written informed consent before initiation of study-specific procedures. The study was conducted in compliance with the Declaration of Helsinki and registered under ClinicalTrials.gov identifier NCT00733850.

\section{Randomization}

Randomization to one of the two treatment groups occurred in a 2:1 (KLTi + gemcitabine: gemcitabine) ratio in this open-label study. The study site obtained the patient's treatment assignment by using a centralized Interactive Voice Response System (IVRS) for Cohort 1 and 2. For Cohort 3, the study site obtained the patient's treatment assignment by using a centralized IWRS (Interactive Web Response System) within DataTrak EDC maintained by Pharm-Olam International, a $3^{\text {rd }}$ party CRO. Randomization was stratified by ECOG status (0/1) or KPS $80-100$ vs. ECOG status 2 or KPS $60-70$ in Cohort 1 and 2, and by site in Cohort 3. For the primary endpoint of PFS, radiologic tumor response was assessed in a blinded manner by a central CT reading facility, BioClinica, Inc. (Princeton, NJ), using RECIST 1.1 criteria.

\section{Procedures}

All patients were to receive marketed gemcitabine. Whenever Kanglaite Injection was administered on the same day as gemcitabine, the KLTi was given first, with gemcitabine followed at least 30 minutes after the end of the KLTi infusion. Gemcitabine was administered intravenously, by peripheral or central line, over a minimum of 30 minutes (and a maximum of 60 minutes), on days 1, 8 and 15 of each 28 day cycle. Kanglaite Injection was administered via a central venous line on days 1-5, $8-12$, and $15-19$ of each 28 day cycle. The total infusion time for $300 \mathrm{~mL}$ of KLTi $(30 \mathrm{gm} / \mathrm{dl})$ in Cohorts 1 and 3 was expected to be approximately 120 minutes. The total infusion time for $500 \mathrm{~mL}$ of KLTi in Cohort 2 was expected to be approximately 180 minutes.

The investigators evaluated the tumor response in patients every 8 weeks until disease progression by means of computed tomography imaging. In addition, all scans were assessed by blinded central radiologic assessment using RECIST 1.1. Patients were followed for survival until death or study closure.

Safety was monitored by means of investigators' assessment of treatment-emergent adverse events and serious adverse events, central laboratory testing and dose delays, interruptions, modifications, and premature discontinuation of the study drug.

\section{Study endpoints/Outcomes}

The primary endpoint was progression-free survival (PFS) by centralized blinded radiologic assessment. Overall survival (OS) and objective response rate (ORR) were secondary endpoints. Patient reported quality of life outcome (Functional Assessment of Anorexia Cachexia Therapy (FAACT) (Cohort 1 \& 2), National Comprehensive Cancer Network, Functional Assessment of Cancer Therapy, Hepatobiliary Symptom Indexes-18 (NCCN-FACT FHSI-18) (Cohort 3)) was also included as a secondary endpoint. Additional efficacy endpoints included disease stabilization rate (defined as complete response, partial response, or stable disease) and 1-year survival rate.

Treatment-emergent adverse events (TEAE) were graded according to the National Cancer Institute Common Terminology Criteria for Adverse Events (NCI-CTCAE), version 4.0 and were coded and summarized using system organ class and preferred terms of Medical Dictionary for Regulatory Activities (MedDRA), version 15.1. The effect of Kanglaite Injection on the time to AEs commonly associated with gemcitabine monotherapy and duration of gemcitabine dosing was investigated.

\section{Statistical analysis}

PFS and overall survival were analyzed with the use of the Kaplan-Meier method and the log-rank test. The hazard ratio and its two-sided 95\% confidence intervals using a Cox regression model were calculated. Sample size was empirically determined in Cohort 1 and 2. Cohort 3 was powered to include an additional 100 patients to provide 74 PFS events to detect a $50 \%$ reduction in the risk of PFS events in the KLTi plus gemcitabine arm, assuming a median PFS of 4 months in the KLTi plus gemcitabine arm, and a median PFS of 2 months in the gemcitabine arm, with 24 months accrual plus 12 months follow-up, 2:1 randomization ratio, a two-sided alpha level of 0.05 , and $80 \%$ power.

A multivariate analysis of survival was performed with the use of a Cox proportional hazard model to evaluate treatment effect with adjustment for baseline imbalance and potential prognostic factors. The comparisons of objective response rate and disease stabilization rate were performed with the use of the Pearson's Chi-square test and exact 95\% Clopper-Pearson CI for point estimate. The difference in 1-year survival was compared using normal approximation and Greenwood's formula for SE. The time to AEs commonly associated with gemcitabine 
monotherapy was investigated with the use of the Kaplan-Meier method, the log-rank test, and the corresponding hazard ratio from Cox regression model. Duration of gemcitabine dosing was explored with the use of an ANCOVA model with treatment as the main effect. T test or Wilcoxon rank sum test was used for quality of life data. All tests were two-sided. There were no adjustments for multiple comparisons, nor was there any splitting of type I error among analyses. SAS version 9.3 was used for analyses.

Efficacy analyses were based on the intent-to-treat population. Adverse events, clinical laboratory data and study drug exposure were analyzed based on the safety population which comprised all patients who received any study drug. Supportive efficacy analyses were provided based on the Efficacy Evaluable (EE) population which consisted of all ITT patients without major protocol deviations, who received at least one dose of study medication, and for whom both baseline and at least one post-treatment tumor assessment were available.

A data monitoring committee (DMC) was established with the responsibility of safe guarding the interests of patients and the DMC reviewed safety data on a regular basis during the conduct of the study.

The study was registered on ClinicalTrials.gov under identifier NCT00733850.

\section{Role of the funding source}

This study was funded and conducted by KangLaiTe USA, Inc.

\section{Results}

\section{Patients and treatment}

A total of 85 patients were randomized in this study. Fifty-three patients received KLTi plus standard doses of gemcitabine, and 27 patients received the standard gemcitabine regimen. Forty-one patients were randomized into Cohort 1 ( $30 \mathrm{~g} /$ day). After a review by the Data Monitoring Committee, an additional 18 patients were randomized into Cohort 2 (50 g/day), but due to feasibility difficulties with enrollment and retention of patients as a result of the long infusion time, Cohort 2 was terminated. A third group, Cohort 3, was added to evaluate an additional 100 patients to KLTi $30 \mathrm{~g} /$ day plus gemcitabine versus gemcitabine monotherapy. Cohort 3 was also stopped early after 26 patients were randomized due to slowed enrollment following the FDA approval of Abraxane on September 6, 2013, to treat patients with late stage pancreatic cancer. Figure 1 provides a schema of patient disposition for each cohort. The first patient was randomized into Cohort 1 on August 25, 2008. The last patient in Cohort 3 was randomized on February 7, 2014.

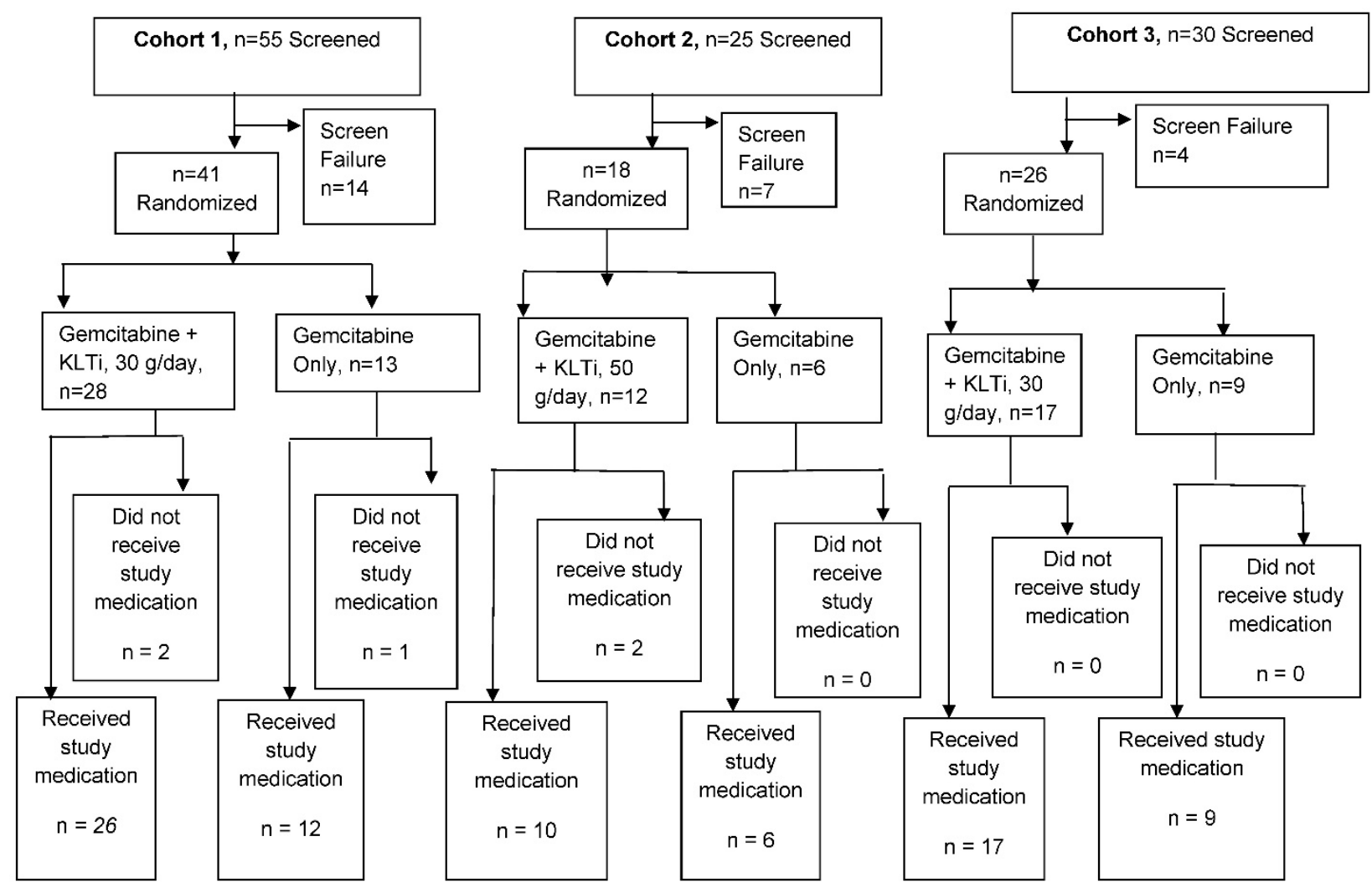

Figure 1: Patient Disposition 


\section{Cohort 1 Results}

Demographic and clinical characteristics at baseline, including key prognostic factors of age, Karnofsky performance status and number of lesions, were generally well balanced between the two arms in Cohort 1 (Table 1). Mean age was 65.6 years in KLTi + gemcitabine versus 65.8 in gemcitabine monotherapy. $89.3 \%$ in KLTi + gemcitabine had stage IV cancer versus $92.3 \%$ in gemcitabine monotherapy. $100 \%$ had prior surgery. Median total number of lesions was 4.5 in $\mathrm{KLTi}+$ gemcitabine versus 5 in gemcitabine monotherapy.

Table 1: Cohort 1-Demographic and Baseline Characteristics (ITT)

\begin{tabular}{lll}
\hline & $\begin{array}{l}\text { KLTi+Gemcitabine } \\
(\mathbf{N = 2 8})\end{array}$ & $\begin{array}{l}\text { Gemcitabine } \\
\mathbf{( N = 1 3 )}\end{array}$ \\
\hline $\begin{array}{l}\text { Age (Years) } \\
\text { Sex }\end{array}$ & $65.6(45-84)$ & $65.8(41-81)$ \\
Male & $12(42.9 \%)$ & $8(61.5 \%)$ \\
Female & $16(57.1 \%)$ & $5(38.5 \%)$ \\
Ethnic Origin & & $1(7.7 \%)$ \\
Asian & 0 & $2(15.4 \%)$ \\
Black & $3(10.7 \%)$ & $2(15.4 \%)$ \\
Hispanic & $3(10.7 \%)$ & $8(61.5 \%)$ \\
White & $22(78.6 \%)$ & 0 \\
Cancer Stage & $1(3.6 \%)$ & $1(7.7 \%)$ \\
IIA & $2(7.1 \%)$ & $12(92.3 \%)$ \\
III & $25(89.3 \%)$ & $1(7.7 \%)$ \\
IV & & $6(46.2 \%)$ \\
Karnofsky Performance Status & $5(38.5 \%)$ \\
70 & $2(7.1 \%)$ & $1(7.7 \%)$ \\
80 & $12(42.9 \%)$ & $13(100 \%)$ \\
90 & $10(35.7 \%)$ & $5(1-11)$ \\
100 & $4(14.3 \%)$ & $0.8(0.1-10.8)$ \\
Number of Total Lesions & & \\
Median (Range) & $4.5(1-15)$ & \\
Duration of Disease (Months) & & \\
Median (Range) & $1.4(0.3-80.2)$ & \\
Prior Surgery & & \\
Yes & & \\
\hline & & \\
\hline
\end{tabular}

\section{Progression-free survival}

The primary efficacy endpoint of progressionfree survival by the independent radiologic assessment in Cohort 1 was calculated using the ITT population. Twenty-three patients (56.1\%) experienced disease progression or died, including $42.9 \%$ in the KLTi + gemcitabine group and $84.6 \%$ in the gemcitabine group. The KLTi + gemcitabine group had a statistically significant improvement in progression-free survival (PFS), with a median of 114 days, versus 57.5 days in the gemcitabine group (HR $0.33 ; 95 \%$ CI: $0.14,0.78), p=0.0080$. At one year, the percentage of patients in Cohort 1 who were progression free was $34.9 \%$ in the KLTi + gemcitabine group versus $8.3 \%$ in the gemcitabine group. Results from 3 PFS sensitivity analyses with different censoring rules for missing scans and post-study anti-cancer therapies were all consistent with the primary analysis. The sensitivity analyses are included in the Supplemental Materials section.

\section{Overall survival}

Overall Survival analysis in Cohort 1 was based on 36 deaths ( $88 \%$ of patients), including 26 in the $\mathrm{KLTi}+$ gemcitabine group (93\%) and 10 in the gemcitabine group $(77 \%)$. In the ITT population, the median survival was 218 days [ $95 \%$ CI, 142 to 325] in the KLTi + gemcitabine group, as compared with 162 days [95\% CI, 39 to 232] in the gemcitabine group (hazard ratio for death was $0.60 ; 95 \%$ CI: $0.28,1.27$ ), p $=0.1758$. At one year, the survival rate in Cohort 1 was $26.9 \%$ in the KLTi + gemcitabine group versus $9.1 \%$ in the gemcitabine group, with a difference of $17.8 \%$ [95\% CI: $-6.2 \%, 41.9 \%$ ], $p=0.1465$. Results from OS sensitivity analyses that censored patients at the start of other anti-cancer therapies were consistent with the primary OS analysis.

\section{Objective Response Rate}

The Objective Response Rate by independent radiologic assessment in the ITT population in Cohort 1 was $14.3 \%(4 / 28)$ in the KLTi + gemcitabine group versus $7.7 \%(1 / 13)$ in the gemcitabine group. Disease stabilization rate $(\mathrm{CR}+\mathrm{PR}+\mathrm{SD})$ in the ITT population was $60.7 \%$ [95\% CI, 40.6 to 78.5 ] in the KLTi + gemcitabine group versus $23.1 \%$ [ $95 \% \mathrm{CI}, 5.0$ to 53.8 ] in the gemcitabine group; $p=0.0249$. Similarly, in the Efficacy Evaluable (EE) population, the Objective Response Rate was $18.2 \%$ in the KLTi + gemcitabine group versus $11.1 \%$ in the gemcitabine group. Disease stabilization rate $(\mathrm{CR}+\mathrm{PR}+\mathrm{SD})$ in the EE population was $77.3 \%$ [ $95 \% \mathrm{CI}, 54.6$ to 92.2] in the KLTi + gemcitabine group versus $33.3 \%$ [ $95 \% \mathrm{CI}, 7.5$ to 70.1 ] in the gemcitabine group; $\mathrm{p}=0.0203$.

\section{Functional Assessment of Cancer Therapy}

Descriptive summary of change from baseline Functional Assessment of Anorexia Cachexia Therapy (FAACT) Total Score, Physical Well-being, Emotional Well-being, Functional Well-being, and Anorexia Cachexia Subscales suggested improved quality of life in Cohort 1 in the $\mathrm{KLTi}+$ gemcitabine group compared to the gemcitabine group. This was observed in all cycles, though it was most noticeable at Cycle 2 as illustrated in the following mean (SD) change from baseline FAACT scores: Total Score of 7.7 (25.46) in the KLTi + gemcitabine group $(\mathrm{n}=14)$ versus -14.6 (17.19) in the gemcitabine group $(\mathrm{n}=7), \mathrm{p}=$ 0.0508; Physical Well-being of 2.4 (6.96) in the KLTi + gemcitabine group $(n=14)$ versus $-4.6(4.59)$ in the gemcitabine group $(n=7), \quad p=0.0269 ; \quad$ Emotional Well-being of 4.0 (5.33) in the KLTi + gemcitabine 
group $(\mathrm{n}=14)$ versus -1.6 (2.94) in the gemcitabine group $(n=7), p=0.0063$; Functional Well-being of 0.3 (5.39) in the KLTi + gemcitabine group $(n=14)$ versus -4.7 (4.81) in the gemcitabine group $(n=7), p=0.0558$; and Anorexia Cachexia Subscale of 2.9 (10.77) in the $\mathrm{KLTi}+$ gemcitabine group $(\mathrm{n}=14)$ versus -4.3 (5.98) in the gemcitabine group $(n=7), p=0.1191$. Positive values indicated improvement.

\section{Cohort 2}

Cohort 2 demographic and baseline characteristics in the ITT population are presented in Table 2. Baseline imbalance was observed in this cohort. Specifically, KLTi +gemcitabine had more patients without prior surgery $(16.7 \%$ vs 0$)$, fewer patients with cancer stage II/III (0 vs $16.7 \%$ in gemcitabine only patients), and fewer patients with 0-1 target lesions at baseline $(8.3 \%$ vs $50 \%$ in gemcitabine only patients). A brief summary of Cohort 2 results are included in the Supplemental Materials section.

Table 2: Cohort 2-Demographic and Baseline Characteristics (ITT)

\begin{tabular}{|c|c|c|}
\hline & $\begin{array}{l}\text { KLTi+Gemcitabine } \\
(\mathrm{N}=12)\end{array}$ & $\begin{array}{l}\text { Gemcitabine } \\
(\mathrm{N}=6)\end{array}$ \\
\hline Age (Years) & $65.7(48-82)$ & $66.7(52-77)$ \\
\hline \multicolumn{3}{|l|}{ Sex } \\
\hline Male & $7(58.3 \%)$ & $1(16.7 \%)$ \\
\hline Female & $5(41.7 \%)$ & $5(83.3 \%)$ \\
\hline \multicolumn{3}{|l|}{ Ethnic Origin } \\
\hline Black & $2(16.7 \%)$ & $1(16.7 \%)$ \\
\hline Hispanic & $1(8.3 \%)$ & 0 \\
\hline Native & 0 & $1(16.7 \%)$ \\
\hline White & $9(75.0 \%)$ & $4(66.7 \%)$ \\
\hline \multicolumn{3}{|l|}{ Cancer Stage } \\
\hline IIB & 0 & $1(16.7 \%)$ \\
\hline III & 0 & 0 \\
\hline IV & $11(91.7 \%)$ & $5(83.3 \%)$ \\
\hline \multicolumn{3}{|c|}{ Karnofsky Performance Status } \\
\hline 70 & 0 & $1(16.7 \%)$ \\
\hline 80 & $3(25.0 \%)$ & 0 \\
\hline 90 & $3(25.0 \%)$ & $2(33.3 \%)$ \\
\hline 100 & $3(25.0 \%)$ & $1(16.7 \%)$ \\
\hline \multicolumn{3}{|c|}{ Number of Total Lesions } \\
\hline Median (Range) & $4.5(1-10)$ & $4(2-8)$ \\
\hline \multicolumn{3}{|c|}{ Duration of Disease (Months) } \\
\hline Median (Range) & $0.8(0.3-6.5)$ & $1.0(0.3-29.8)$ \\
\hline \multicolumn{3}{|l|}{ Prior Surgery } \\
\hline Yes & $10(83.3 \%)$ & $6(100 \%)$ \\
\hline
\end{tabular}

\section{Cohort 1+3 Results}

The patient population enrolled in Cohort 3 appeared to vary over time, with differences seen in the median overall survival of 100 days among post Abraxane approval patients $(\mathrm{n}=16)$, versus a median overall survival of 192 days among pre Abraxane approval patients $(\mathrm{n}=10)$ in this cohort. Cohort 3 demographic and baseline characteristics in the ITT population are presented in Table 3. Endpoint results for cohort 3 are available in the supplemental information. The primary and secondary endpoints reflect the combined Cohort $1+3$.

Table 3: Cohort 3-Demographic and Baseline Characteristics (ITT)

\begin{tabular}{|c|c|c|}
\hline & $\begin{array}{l}\text { KLTi+Gemcitabine } \\
(\mathrm{N}=17)\end{array}$ & $\begin{array}{l}\text { Gemcitabine } \\
(\mathrm{N}=9)\end{array}$ \\
\hline Age (Years) & $63.9(33-79)$ & $63.9(44-81)$ \\
\hline \multicolumn{3}{|l|}{ Sex } \\
\hline Male & $8(47.1 \%)$ & $4(44.4 \%)$ \\
\hline Female & $9(52.9 \%)$ & $5(55.6 \%)$ \\
\hline \multicolumn{3}{|l|}{ Ethnic Origin } \\
\hline Black & $1(5.9 \%)$ & $4(44.4 \%)$ \\
\hline Hispanic & $3(17.6 \%)$ & $2(22.2 \%)$ \\
\hline White & $13(76.5 \%)$ & $3(33.3 \%)$ \\
\hline \multicolumn{3}{|l|}{ Cancer Stage } \\
\hline III & $2(11.8 \%)$ & 0 \\
\hline IV & $15(88.2 \%)$ & $9(100 \%)$ \\
\hline \multicolumn{3}{|c|}{ Karnofsky Performance Status } \\
\hline 60 & $2(11.8 \%)$ & 0 \\
\hline 70 & $1(5.9 \%)$ & $1(11.1 \%)$ \\
\hline 80 & $6(35.3 \%)$ & $4(44.4 \%)$ \\
\hline 90 & $5(29.4 \%)$ & $3(33.3 \%)$ \\
\hline 100 & $3(17.6 \%)$ & $1(11.1 \%)$ \\
\hline \multicolumn{3}{|c|}{ Number of Total Lesions } \\
\hline Median (Range) & $4(1-6)$ & $5(4-7)$ \\
\hline \multicolumn{3}{|c|}{ Duration of Disease (Months) } \\
\hline Median (Range) & $0.9(0.3-38.2)$ & $0.6(0.2-2.3)$ \\
\hline \multicolumn{3}{|l|}{ Prior Surgery } \\
\hline Yes & $7(41.2 \%)$ & $5(55.6 \%)$ \\
\hline No & $10(58.8 \%)$ & $4(44.4 \%)$ \\
\hline
\end{tabular}

\section{Progression-free survival}

In the progression-free survival based on the independent radiologic assessment (ITT population) among Cohort 1 and 3 patients, 44 patients (65.7\%) had disease progression or died, including $55.6 \%$ in the KLTi + gemcitabine group and $86.4 \%$ in the gemcitabine group. The KLTi + gemcitabine group had a statistically significant improvement in progression-free survival (PFS), with a median of 112 days, compared to a median PFS of 58 days in the gemcitabine group. HR from Cohort $1+3$ pooled analysis was $0.50 ; 95 \% \mathrm{CI}: 0.27,0.92, \mathrm{p}=0.0240$. HR from Cox regression stratified by cohort was $0.53,95 \%$ CI: $0.28,0.99, \mathrm{p}=0.0421$. HR from Cox regression stratified by cohort, was $0.57,95 \%$ CI: $0.28,1.16$ after adjustment for baseline imbalance and prognostic factors that included baseline disease duration, total number of lesions, race, Karnofsky Performance Status, and age. At one year, the percentage of patients in Cohort $1+3$ who were progression free was $18.5 \%$ in the KLTi + gemcitabine group versus $0 \%$ in the gemcitabine group. Results from 3 PFS sensitivity analyses with different censoring rules for missing scans and post-study anti-cancer therapies were all consistent with the primary analysis. 


\section{Overall survival}

Cohort $1+3$ Overall Survival analysis was based on 56 deaths (83.6\% of patients), including 38 in the $\mathrm{KLTi}+$ gemcitabine group $(84.4 \%)$ and 18 in the gemcitabine group $(81.8 \%)$. In the ITT population, the median survival was 178 days [ $95 \% \mathrm{CI}, 118$ to 274] in the KLTi + gemcitabine group, as compared with 162 days [95\% CI, 74 to 195] in the gemcitabine group (hazard ratio for death in the pooled analysis was $0.68 ; 95 \%$ CI: $0.38,1.21), p=0.1830$. When stratified by cohort, the hazard ratio for death in the pooled analysis was 0.73 ; 95\% CI: 0.40, 1.31. When stratified by cohort and adjusted for imbalance in baseline prognostic factors of disease duration, total number of lesions, race, and Karnofsky Performance Status, the hazard ratio for death in the pooled analysis was 0.81 ; 95\% CI: 0.40, 1.64. Cohort 3 patients with last known alive date post study cut-off date were censored on 13JUN2014. At one year, the survival rate in Cohort $1+3$ was $24.3 \%$ in the KLTi + gemcitabine group versus $5.5 \%$ in the gemcitabine group, with a difference of $18.8 \%$ [95\% CI: $1.8 \%, 35.8 \%$ ], $p=0.0302$. Results from OS sensitivity analyses that censored patients at the start of other anti-cancer therapies were consistent with the primary OS analysis.

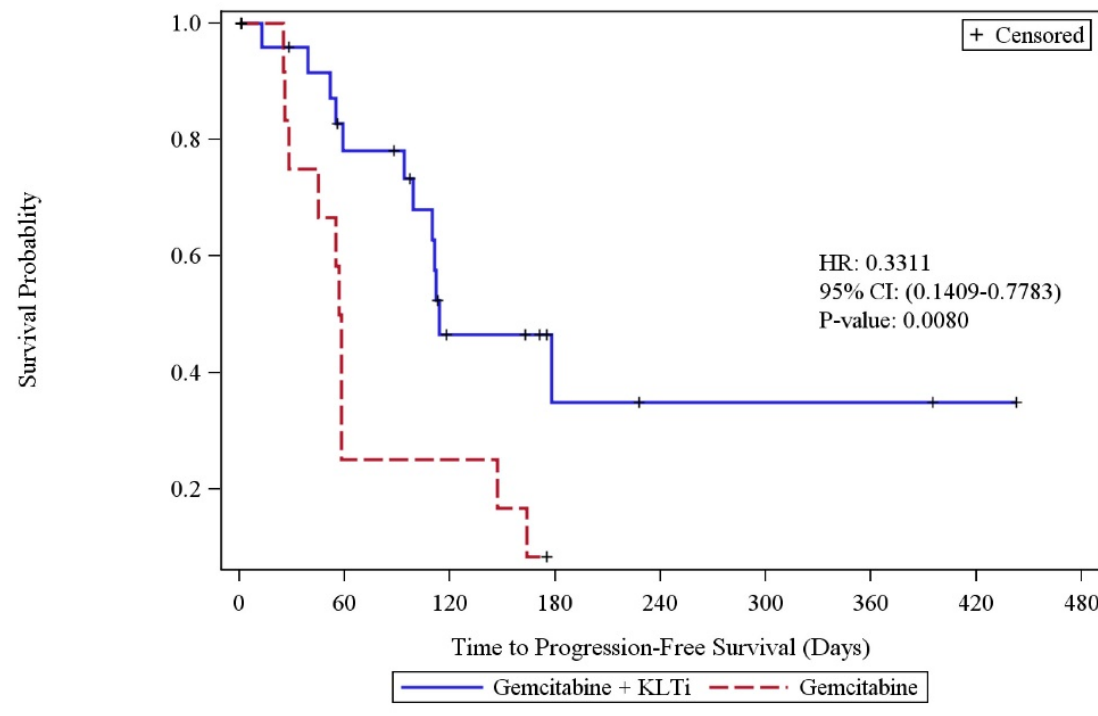

Number at Risk:

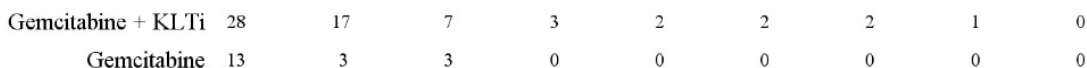

Figure 2: Cohort 1-Kaplan-Meier Plot of Progression-Free Survival by Radiologic Assessment (ITT Population)

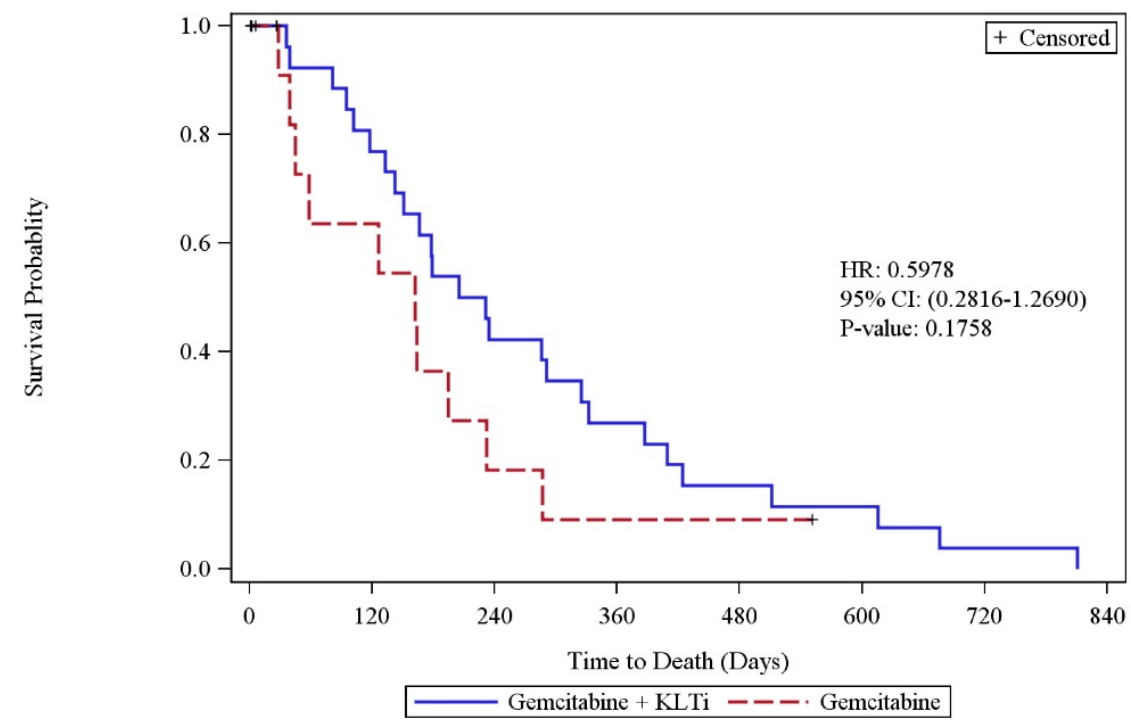

Number at Risk:

Gemcitabine + KLTi

Gemcitabine 13

$\begin{array}{ccccccc}20 & 11 & 7 & 4 & 3 & 1 & 0 \\ 7 & 2 & 1 & 1 & 0 & 0\end{array}$

Figure 3: Kaplan-Meier Plot of Overall Survival (ITT Population) 


\section{Objective Response Rate}

The Objective Response Rate by independent radiologic assessment in the ITT population in Cohort $1+3$ was $15.6 \%(7 / 45)$ in the KLTi + gemcitabine group versus $4.5 \%(1 / 22)$ in the gemcitabine group. The odds ratio from Cochran-Mantel-Haenszel test stratified by cohort was 3.77, 95\% CI: 0.44, 32.23, $\mathrm{p}=0.1991$. Disease stabilization rate $(C R+P R+S D)$ in the ITT population was $55.6 \%(25 / 45)$ in the KLTi + gemcitabine group versus $36.4 \%(8 / 22)$ in the gemcitabine group. The odds ratio from Cochran-Mantel-Haenszel test stratified by cohort was $2.12,95 \%$ CI: 0.76, 5.94, p=0.1451. Similarly, in the EE population, the Objective Response Rate was $21.2 \%(7 / 33)$ in the KLTi + gemcitabine group versus $5.6 \%(1 / 18)$ in the gemcitabine group, $p=0.1488$. Disease stabilization rate $(\mathrm{CR}+\mathrm{PR}+\mathrm{SD})$ in the $\mathrm{EE}$ population was $75.8 \%(25 / 33)$ in the KLTi + gemcitabine group versus $44.4 \%(8 / 18)$ in the gemcitabine group; $\mathrm{p}=0.0260$.

\section{Functional Assessment of Cancer Therapy}

Due to the fact that the Functional Assessment of Anorexia Cachexia Therapy (FAACT) was utilized for Cohort 1 and 2, and the Functional Assessment of Cancer Therapy, Hepatobiliary Symptom Indexes-18 (FACT FHSI-18) was utilized for Cohort 3, no analysis of descriptive summary is possible for the combined Cohort $1+3$. Cohort 3 summary is presented separately. Descriptive summary of change from baseline FACT FHSI-18 Total Score and Disease Related Symptoms - Physical Subscale in the ITT population suggested improved quality of life in Cohort 3 in the KLTi + gemcitabine group compared to the gemcitabine group. This was most noticeable at Cycle 1 Day 19: mean (SD) change from baseline FACT FHSI-18 Total Score of 0.5 (8.30) in the KLTi + gemcitabine group $(n=13)$ versus $-10.3(5.23)$ in the gemcitabine group $(n=6), p=0.0099$; and Disease Related Symptoms - Physical of 0.9 (5.01) in the KLTi + gemcitabine group $(n=13)$ versus -8.0 (4.19) in the gemcitabine group $(n=6), p=0.0015$. Positive values indicated improvement. All $\mathrm{p}$-values provided are considered nominal and are for descriptive purposes only.

\section{Treatment-emergent Adverse Events}

Overall, among all 80 patients who received study treatment in the study, the incidence rates of TEAEs, TEAEs of NCI-CTCAE Grade 3 or higher, SAEs and AEs leading to study treatment discontinuation were similar between the two treatment groups. A total of $47 / 53(88.7 \%)$ patients who received KLTi + gemcitabine and 24/27 (88.9\%) of patients who received gemcitabine had at least 1
Grade 3 or higher TEAEs. A total of 31/53 (58.5\%) patients who received KLTi + gemcitabine and 15/27 (55.6\%) of patients who received gemcitabine had serious adverse events (SAEs). A total of 17/53 (32.1\%) patients who received KLTi + gemcitabine and $8 / 27(29.6 \%)$ of patients who received gemcitabine reported at least 1 adverse event leading to study treatment discontinuation. There were no deaths related to treatment with KLTi + gemcitabine. Table 3 provides a by treatment and by grade summary for TEAEs with incidence $>=10 \%$ in either treatment.

The time to occurrence of the most common $(\geq 5 \%)$ Grade 3 or above TEAEs (neutropenia, nausea/vomiting, increased ALT, increased alkaline phosphatase, anemia, increased AST, and thrombocytopenia, (as reported in the Gemzar Package Insert) was significantly delayed in the KLTi + gemcitabine group $(\mathrm{N}=31)$ compared with the gemcitabine alone group $(\mathrm{N}=13)(\mathrm{p}=0.0436$; HR $[95 \% \mathrm{CI}]=0.4661[0.2192,0.9912]$ ) (Figure 6).

Among the 80 patients in the Safety population, treatment duration was 24 days longer in KLTi + gemcitabine group compared to the gemcitabine only group $(p=0.1494)$, which reflected one additional cycle of therapy. Among patients with grade 3 or above neutropenia $(\mathrm{N}=22)$, an increase of 48 days in gemcitabine treatment duration in $\mathrm{KLTi}+$ gemcitabine group was observed reflecting approximately 2 additional cycles of gemcitabine dosing. Among patients with neutropenia (all grades) $(\mathrm{N}=28)$, an increase of 38 days in gemcitabine treatment duration in KLTi +gemcitabine group was observed.

\section{Discussion}

Current treatment for locally advanced/metastatic pancreatic cancer remains sub-optimal. Single agent gemcitabine was the mainstay of treatment for many years with median survival of about six months. Recently, the combination therapy FOLFIRINOX has demonstrated survival benefit in select patients with excellent performance status, representing a minority of patients with advanced disease (6). The addition of nab-paclitaxel to gemcitabine led to a statistically significant but clinically small improvement in overall survival, 1.8 months, in a phase 3 trial(7). New approaches are clearly needed.

KangLaiTe injection (KLTi) has been evaluated preclinically for antitumor effects. In pancreatic cell lines, KLT downregulated expression of BCL-2 protein, increased Fas gene expression and increased apoptosis (15). Apoptosis may also be enhanced through stimulation of Caspase-3 induction, seen after 
prolonged exposure to human pancreatic cells to KLTi $(16,17)$. KLTi increases apoptosis in a variety of other tumor cell lines and animal models (18-21).

Recently KLTi was assessed in pancreatic cancer xenografts in mice using the human pancreatic cell line PANC-1(10). After establishment of subcutaneous tumor, treatment with KLTi leads to a statistically significant dose-dependent reduction in tumor volume and weight, up to $85 \%$ reduction. Apoptosis measured by TUNEL assay increased in a doses dependent manner. Evaluation of the $\mathrm{PI} 3 \mathrm{~K} / \mathrm{AKT} / \mathrm{mTOR}$ pathway showed no change in mRNA expression level by KLTi, but a significant reduction in phosphoAKT and phosphor mTOR levels $(p<0.05)$ suggesting interference with pathway activation.

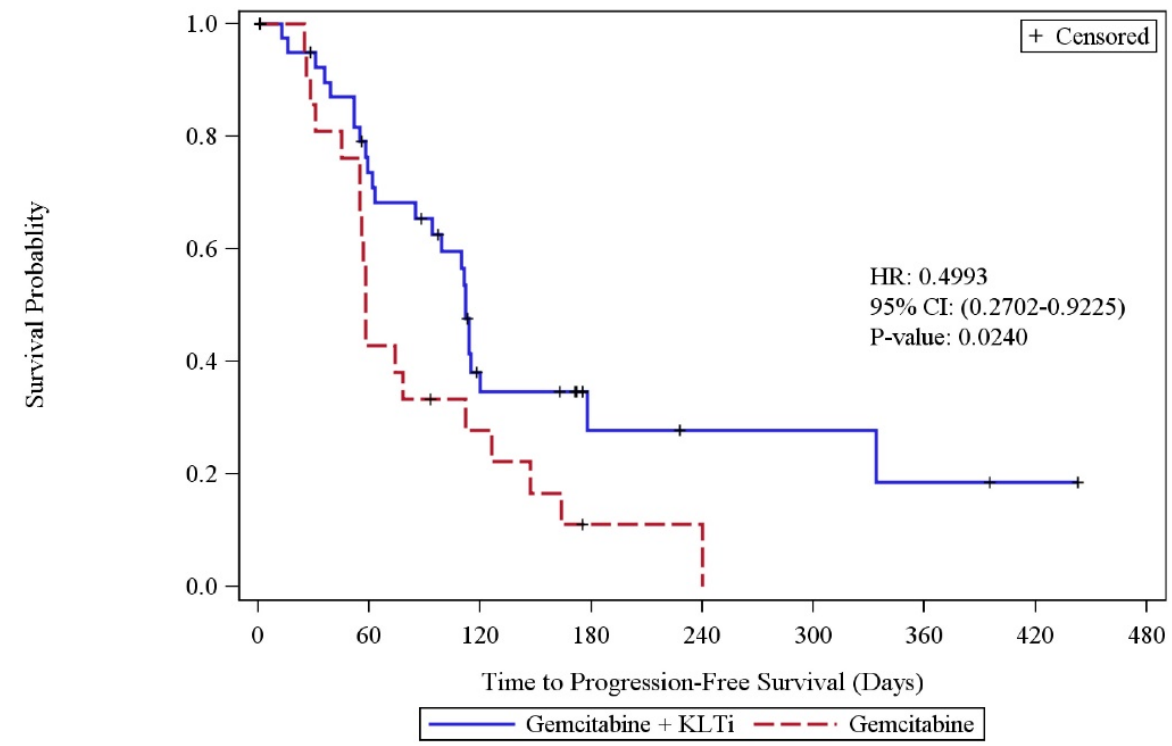

Number at Risk:

$\begin{array}{llllllllll}\text { Gemcitabine + KLTi } & 45 & 27 & 10 & 4 & 3 & 3 & 2 & 1 & 0\end{array}$

$\begin{array}{llllllllll}\text { Gemcitabine } & 22 & 9 & 5 & 1 & 0 & 0 & 0 & 0 & 0\end{array}$

Figure 4: Cohort 1+3-Kaplan-Meier Plot of Progression-Free Survival by Radiologic Assessment (ITT Population)

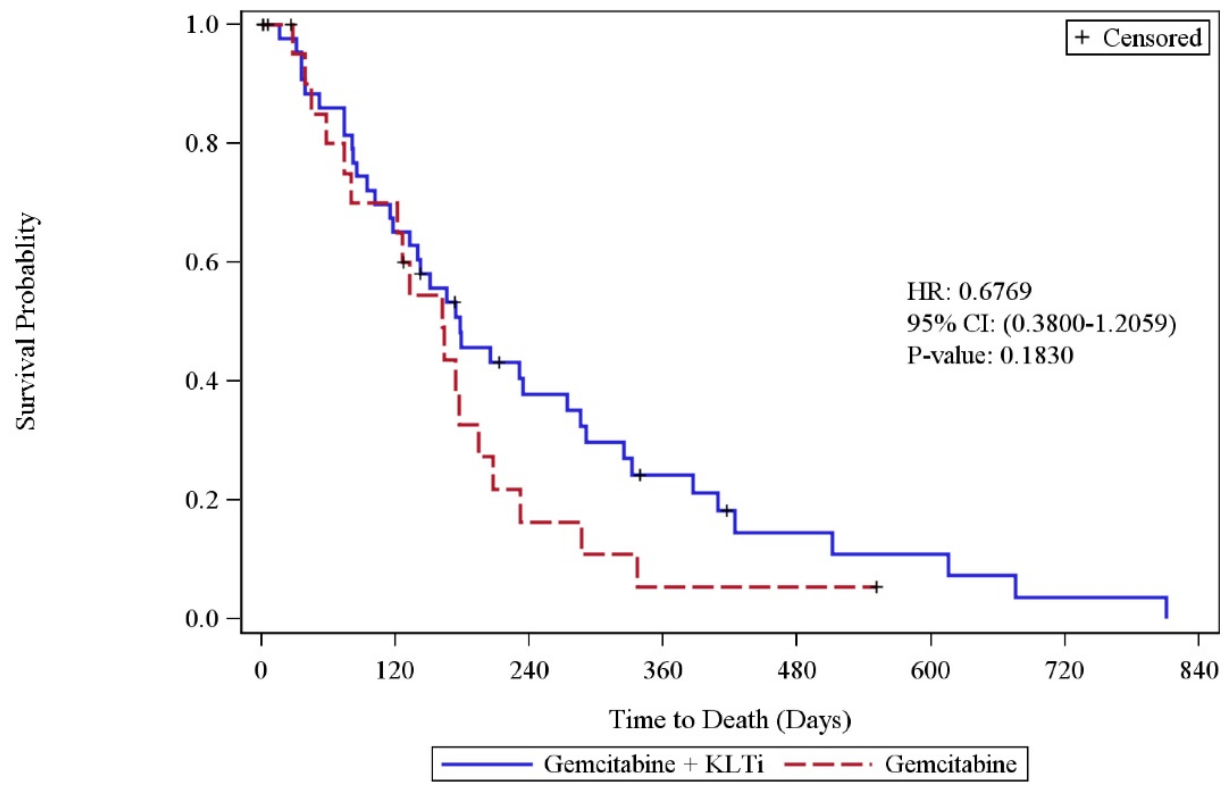

Number at Risk:

Gemcitabine + KLTi 45

Gemcitabine 22

$28 \quad 14$

14

14
3

8

8
1

Figure 5: Cohort 1+3 Kaplan-Meier Plot of Overall Survival (ITT Population) 


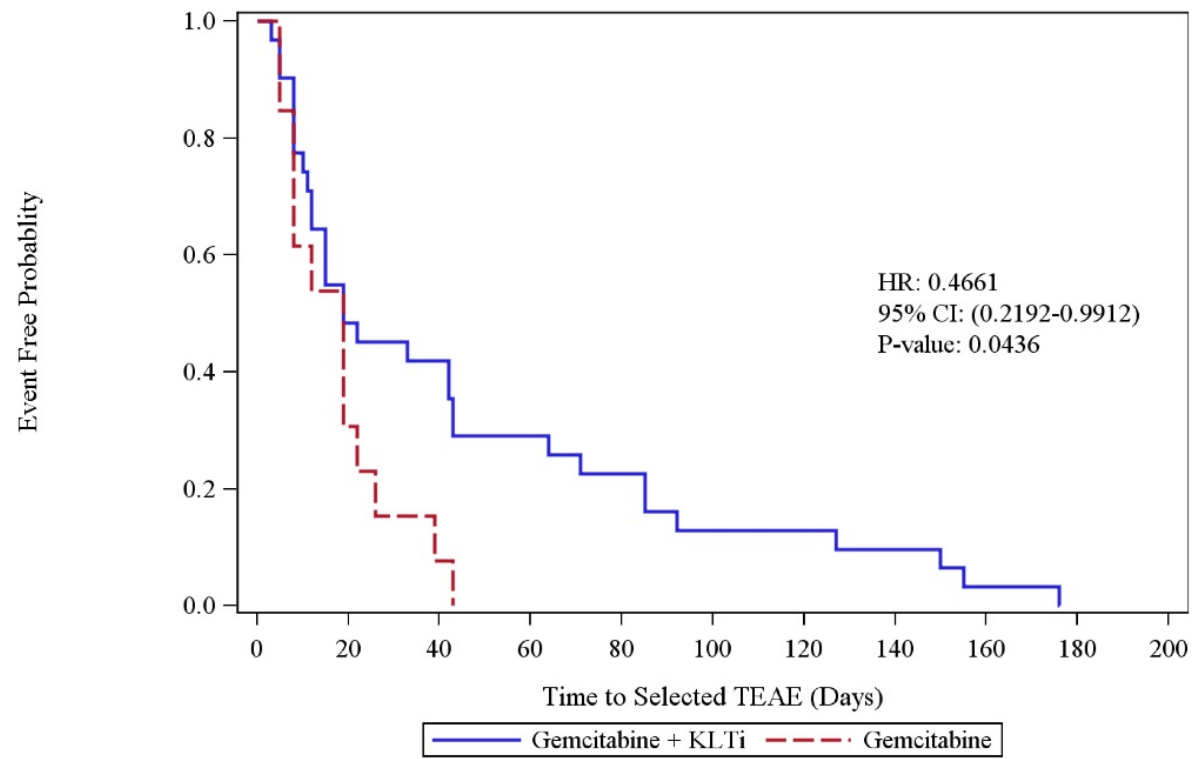

Number at Risk:

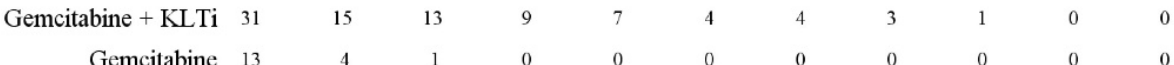

Figure 6: Time to the Most Common Grade 3 or Above TEAEs Associated with Gemcitabine Monotherapy

Table 4: SUMMARY OF TEAES WITH INCIDENCE >= 10\% (SAFETY POPULATION), COHORT $1+2+3$

\begin{tabular}{|c|c|c|c|c|c|c|c|c|}
\hline MedDRA Preferred Term & $\begin{array}{l}\text { KLTi/Gemc } \\
(\mathrm{N}=53)\end{array}$ & abine & & & $\begin{array}{l}\text { Gemcitabin } \\
(\mathrm{N}=27)\end{array}$ & & & \\
\hline & Grade 1-2 & Grade 3-4 & Grade 5 & Overall & Grade 1-2 & Grade 3-4 & Grade 5 & Overall \\
\hline ANAEMIA & $26(49.1 \%)$ & $13(24.6 \%)$ & 0 & $39(73.6 \%)$ & $10(37.0 \%)$ & $7(25.9 \%)$ & 0 & $17(63.0 \%)$ \\
\hline NAUSEA & $29(54.7 \%)$ & $2(3.8 \%)$ & 0 & $31(58.5 \%)$ & $13(48.1 \%)$ & $2(7.4 \%)$ & 0 & $15(55.6 \%)$ \\
\hline FATIGUE & $20(37.7 \%)$ & $4(7.5 \%)$ & 0 & 24 (45.3\%) & $7(25.9 \%)$ & $6(22.2 \%)$ & 0 & $13(48.1 \%)$ \\
\hline OEDEMA PERIPHERAL & $20(37.8 \%)$ & $3(5.7 \%)$ & 0 & $23(43.4 \%)$ & $12(44.4 \%)$ & 0 & 0 & $12(44.4 \%)$ \\
\hline PYREXIA & $20(37.8 \%)$ & $2(3.8 \%)$ & 0 & $22(41.5 \%)$ & $6(22.2 \%)$ & 0 & 0 & $6(22.2 \%)$ \\
\hline VOMITING & $19(35.8 \%)$ & $2(3.8 \%)$ & 0 & $21(39.6 \%)$ & $9(33.3 \%)$ & $3(11.1 \%)$ & 0 & $12(44.4 \%)$ \\
\hline THROMBOCYTOPENIA & $14(26.4 \%)$ & $6(11.4 \%)$ & 0 & $20(37.7 \%)$ & $11(40.7 \%)$ & $1(3.7 \%)$ & 0 & $12(44.4 \%)$ \\
\hline ABDOMINAL PAIN & $10(18.8 \%)$ & $9(17.0 \%)$ & 0 & $19(35.8 \%)$ & $3(11.1 \%)$ & $4(14.8 \%)$ & 0 & $7(25.9 \%)$ \\
\hline NEUTROPENIA & $4(7.5 \%)$ & $15(28.3 \%)$ & 0 & $19(35.8 \%)$ & $2(7.4 \%)$ & $7(25.9 \%)$ & 0 & $9(33.3 \%)$ \\
\hline DECREASED APPETITE & $17(32.1 \%)$ & $1(1.9 \%)$ & 0 & $18(34.0 \%)$ & $6(22.2 \%)$ & $3(11.1 \%)$ & 0 & $9(33.3 \%)$ \\
\hline CONSTIPATION & $15(28.3 \%)$ & $1(1.9 \%)$ & 0 & $16(30.2 \%)$ & $10(37.0 \%)$ & $1(3.7 \%)$ & 0 & $11(40.7 \%)$ \\
\hline DIARRHOEA & $15(28.3 \%)$ & $1(1.9 \%)$ & 0 & $16(30.2 \%)$ & $8(29.6 \%)$ & 0 & 0 & $8(29.6 \%)$ \\
\hline HYPOTENSION & $12(22.6 \%)$ & $4(7.6 \%)$ & 0 & $16(30.2 \%)$ & $1(3.7 \%)$ & 0 & 0 & $1(3.7 \%)$ \\
\hline HYPOKALAEMIA & $10(18.9 \%)$ & $5(9.4 \%)$ & 0 & $15(28.3 \%)$ & $1(3.7 \%)$ & $2(7.4 \%)$ & 0 & $3(11.1 \%)$ \\
\hline $\begin{array}{l}\text { BLOOD ALKALINE PHOSPHATASE } \\
\text { INCREASED }\end{array}$ & $12(22.6 \%)$ & $2(3.8 \%)$ & 0 & $14(26.4 \%)$ & $2(7.4 \%)$ & $1(3.7 \%)$ & 0 & $3(11.1 \%)$ \\
\hline DEHYDRATION & $6(11.4 \%)$ & $7(13.2 \%)$ & 0 & $13(24.5 \%)$ & $2(7.4 \%)$ & $1(3.7 \%)$ & 0 & $3(11.1 \%)$ \\
\hline $\begin{array}{l}\text { ALANINE AMINOTRANSFERASE } \\
\text { INCREASED }\end{array}$ & $9(17.0 \%)$ & $3(5.7 \%)$ & 0 & $12(22.6 \%)$ & $2(7.4 \%)$ & $3(11.1 \%)$ & 0 & $5(18.5 \%)$ \\
\hline ASTHENIA & $9(17.0 \%)$ & $3(5.7 \%)$ & 0 & $12(22.6 \%)$ & $3(11.1 \%)$ & $2(7.4 \%)$ & 0 & $5(18.5 \%)$ \\
\hline BACK PAIN & $8(15.1 \%)$ & $3(5.7 \%)$ & 0 & $11(20.8 \%)$ & $3(11.1 \%)$ & $3(11.1 \%)$ & 0 & $6(22.2 \%)$ \\
\hline DYSPNOEA & $9(17.0 \%)$ & $2(3.8 \%)$ & 0 & $11(20.8 \%)$ & $1(3.7 \%)$ & $2(7.4 \%)$ & 0 & $3(11.1 \%)$ \\
\hline $\begin{array}{l}\text { ASPARTATE } \\
\text { AMINOTRANSFERASE } \\
\text { INCREASED }\end{array}$ & $7(13.2 \%)$ & $3(5.7 \%)$ & 0 & $10(18.9 \%)$ & $3(11.1 \%)$ & $1(3.7 \%)$ & 0 & $4(14.8 \%)$ \\
\hline BLOOD BILIRUBIN INCREASED & $4(7.5 \%)$ & $6(11.3 \%)$ & 0 & $10(18.9 \%)$ & 0 & $3(11.1 \%)$ & 0 & $3(11.1 \%)$ \\
\hline DISEASE PROGRESSION & 0 & 0 & $10(18.9 \%)$ & $10(18.9 \%)$ & $1(3.7 \%)$ & $2(7.4 \%)$ & $3(11.1 \%)$ & $6(22.2 \%)$ \\
\hline HYPOALBUMINAEMIA & $9(17.0 \%)$ & $1(1.9 \%)$ & 0 & $10(18.9 \%)$ & 0 & 0 & 0 & 0 \\
\hline ANXIETY & $8(15.1 \%)$ & $1(1.9 \%)$ & 0 & $9(17.0 \%)$ & $3(11.1 \%)$ & 0 & 0 & $3(11.1 \%)$ \\
\hline DEPRESSION & $9(17.0 \%)$ & 0 & 0 & $9(17.0 \%)$ & $2(7.4 \%)$ & 0 & 0 & $2(7.4 \%)$ \\
\hline HYPERGLYCAEMIA & $6(11.3 \%)$ & $3(5.7 \%)$ & 0 & $9(17.0 \%)$ & $3(11.1 \%)$ & $1(3.7 \%)$ & 0 & $4(14.8 \%)$ \\
\hline CELLULITIS & $6(11.3 \%)$ & $2(3.8 \%)$ & 0 & $8(15.1 \%)$ & $1(3.7 \%)$ & $1(3.7 \%)$ & 0 & $2(7.4 \%)$ \\
\hline ABDOMINAL DISTENSION & $7(13.2 \%)$ & 0 & 0 & $7(13.2 \%)$ & $1(3.7 \%)$ & 0 & 0 & $1(3.7 \%)$ \\
\hline DIZZINESS & $6(11.3 \%)$ & $1(1.9 \%)$ & 0 & $7(13.2 \%)$ & $4(14.8 \%)$ & 0 & 0 & $4(14.8 \%)$ \\
\hline
\end{tabular}




\begin{tabular}{|c|c|c|c|c|c|c|c|c|}
\hline HYPOCALCAEMIA & $7(13.2 \%)$ & 0 & 0 & $7(13.2 \%)$ & $1(3.7 \%)$ & 0 & 0 & $1(3.7 \%)$ \\
\hline RASH & $6(11.3 \%)$ & $1(1.9 \%)$ & 0 & $7(13.2 \%)$ & $3(11.1 \%)$ & 0 & 0 & $3(11.1 \%)$ \\
\hline ABDOMINAL PAIN UPPER & $6(11.3 \%)$ & 0 & 0 & $6(11.3 \%)$ & $1(3.7 \%)$ & 0 & 0 & $1(3.7 \%)$ \\
\hline HYPONATRAEMIA & $5(9.4 \%)$ & $1(1.9 \%)$ & 0 & $6(11.3 \%)$ & $3(11.1 \%)$ & $2(7.4 \%)$ & 0 & $5(18.5 \%)$ \\
\hline INSOMNIA & $6(11.3 \%)$ & 0 & 0 & $6(11.3 \%)$ & $2(7.4 \%)$ & $1(3.7 \%)$ & 0 & $3(11.1 \%)$ \\
\hline PAIN IN EXTREMITY & $6(11.3 \%)$ & 0 & 0 & $6(11.3 \%)$ & $3(11.1 \%)$ & 0 & 0 & $3(11.1 \%)$ \\
\hline HEADACHE & $5(9.4 \%)$ & 0 & 0 & $5(9.4 \%)$ & $3(11.1 \%)$ & 0 & 0 & $3(11.1 \%)$ \\
\hline URINARY TRACT INFECTION & $3(5.7 \%)$ & $2(3.8 \%)$ & 0 & $5(9.4 \%)$ & $2(7.4 \%)$ & $2(7.4 \%)$ & 0 & $4(14.8 \%)$ \\
\hline ASCITES & $2(3.8 \%)$ & $2(3.8 \%)$ & 0 & $4(7.5 \%)$ & $2(7.4 \%)$ & $3(11.1 \%)$ & 0 & $5(18.5 \%)$ \\
\hline DEEP VEIN THROMBOSIS & 0 & $4(7.5 \%)$ & 0 & $4(7.5 \%)$ & $1(3.7 \%)$ & $2(7.4 \%)$ & 0 & $3(11.1 \%)$ \\
\hline WEIGHT DECREASED & $4(7.5 \%)$ & 0 & 0 & $4(7.5 \%)$ & $4(14.8 \%)$ & 0 & 0 & $4(14.8 \%)$ \\
\hline DRY MOUTH & $3(5.7 \%)$ & 0 & 0 & $3(5.7 \%)$ & $4(14.8 \%)$ & 0 & 0 & $4(14.8 \%)$ \\
\hline DYSGEUSIA & $3(5.7 \%)$ & 0 & 0 & $3(5.7 \%)$ & $3(11.1 \%)$ & 0 & 0 & $3(11.1 \%)$ \\
\hline
\end{tabular}

Note: [1] Grade: 1 = Mild, 2 = Moderate, 3 = Severe, 4 = Life Threatening, $5=$ Fatal.

KLTi has been widely used clinically in China as an adjunct to chemotherapy since 1995 when phase III trials were completed and the product was marketed. Since that time, over 1 million cancer patients have received KLTi. A meta-analysis of its use in non-small cell lung cancer was published in 2008 by the Chinese Cochrane Evidence-Based Medicine Center (22). This analysis included 26 trials of chemotherapy with or without KLTi in 2209 patients; most studies had moderate or high risk of bias according to the quality assessment. The meta-analysis demonstrated that chemotherapy + KLTi improved response rates (relative risk 1.34 (95\% Confidence Interval, 1.19-1.51) and Karnofsky Performance Status (RR 2.05, 95\% CI 1.60-2.64). Additionally, KLTi reduced adverse events including leukopenia, anemia, thrombocytopenia, nausea and vomiting, and hepatic dysfunction.

Clinical trials in GI cancers adding KLTi to chemotherapy have been conducted. A randomized trial with or without KLTi in sixty patients with advanced gastric cancer receiving chemotherapy was recently reported (23). KLTi was given daily at up to $100 \mathrm{ml} /$ day. The reponse rate (all partial responses) was $40 \%$ in the KLTi arm compared to $16.7 \%$ in the chemotherapy alone group $(\mathrm{p}<0.05)$, and quality of life improved more in the KLTi arm as well $(\mathrm{P}<, 0.05)$. Adverse events were numerically less in the experimental arm. An open-label trial of gemcitabine +/- KLTi was conducted in Chinese pancreatic cancer patients. This study demonstrated an increase of median overall survival of 2.8 months among the 40 patients received combination therapy compared to gemcitabine monotherapy alone in 18 patients (14).

We performed this multi-centered, randomized phase 2 trial to investigate the effects of adding KLTi to gemcitabine in locally advanced/metastatic pancreas cancer. The results demonstrated a significant improvement in progression-free survival, the primary endpoint of the trial, when KLTi 30gm was administered daily $\times 5$ intravenously on days $1-5$, 8-12 and 15-19 of each cycle. KLTi in combination with gemcitabine was well tolerated with a safety profile similar to gemcitabine alone. The study enrolled patients in three cohorts over a 6 year period. Cohort 1 and 3 received 30 gm KLTi per dose while cohort 2 revealed $50 \mathrm{gm}$ KLTi per dose.

In Cohort 1, superior clinical benefits of KLTi + gemcitabine compared with gemcitabine alone were also observed in improved median OS, higher overall response rates as well as improved quality of life including physical well-being, functional well-being, emotional well-being, anorexia and cachexia subscale, and overall quality of life.

Cohort 3 was terminated prematurely due to slowing of patient accrual. Changes in standard of care during the time course of the study may have influenced patient referral and outcomes. Patients in cohort 3 displayed substantially and significantly different baseline characteristics compared to cohort 1 , likely reflecting the changing landscape of standard treatment of advanced pancreatic cancer during this time. Median survival of patients was 100 days among the 16 post-Abraxane approval patients in Cohort 3, versus 179 days observed among the 41 patients in Cohort 1 (HR=0.44 in favor of Cohort 1, 95\% CI: 0.21, 0.93, $\mathrm{p}=0.0158$ ), and 192 days among the 10 pre Abraxane patients in Cohort 3 period suggesting referral bias for perceived poorer outcome patients post- FDA approval of the nab-paclitaxel-gemcitabine combination.

KLTi appeared to have positive effects upon quality of life. In both cohorts, the global QOL as well as physical subscale using well established instruments suggested improvement during treatment with KLTi versus worsening with gemcitabine alone. Similar results have been seen on multiple cancer-related symptoms when KLTi has been utilized in combination with chemotherapy ${ }^{(24)}$.

Adverse events were generally similar between both arms of the study across cohorts. Of special interest, the median time to occurrence of Grade 3 or greater neutropenia was 42 days in the KLTi + gemcitabine group $(\mathrm{N}=15)$ compared with 26 days in the gemcitabine alone group $(\mathrm{N}=7), \mathrm{HR}=0.59$. A 
similar trend was observed in neutropenia (all Grades) and thrombocytopenia (all Grades, and Grades 3 or above). Reduction in hematologic toxicity has also been noted when the KLTi was added to chemotherapy for NSCLC (22). In summary, Kanglaite injection $30 \mathrm{gm}$ added to standard dose gemcitabine improved PFS and demonstrated numerically better one year and median overall survival compared to gemcitabine alone for pancreatic cancer in this randomized phase 2 trial. No safety signals were identified with the addition of the agent and QOL was improved with the addition of KLTi. Given the lack of incremental toxicity and potential attenuation of hematologic toxicity, KLTi could also potentially be added to a combination chemotherapy regimen such as nab-paclitaxel/gemcitabine. Additional trial designs have been explored and a phase 3 trial is being prepared to define the benefit of Kanglaite injection in pancreatic cancer.

\section{Supplementary Material}

Supplemental information.

http://www.jcancer.org/v08p1872s1.pdf

\section{Acknowledgments}

We would like to express our gratitude to the patients who participated in this trial as well as their families. We also thank all sites and investigators on behalf of KangLaiTe USA, Inc. for their participation in this study.

Funding: This study was funded and conducted by KangLaiTe USA, Inc.

\section{Competing Interests}

Lee Schwartzberg and Francis Arena have received consulting fees from KangLaiTe USA, Inc. Mary Tagliaferri and Michael Chen are former employees of KangLaiTe USA, Inc. J. Paul Waymack is a consultant of KangLaiTe USA, Inc. Dapeng Li is an employee of KangLaiTe USA, Inc. The authors have declared that no other competing interest exists.

\section{References}

1. Siegel RL, Miller KD, Jemal A. Cancer statistics, 2016. CA Cancer J Clin. 2016;66(1):7-30. doi: 10.3322/caac.21332. Epub 2016 Jan 7.

2. Sun $\mathrm{H}$, Ma H, Hong $\mathrm{G}$, Sun $\mathrm{H}$, Wang J. Survival improvement in patients with pancreatic cancer by decade: a period analysis of the SEER database, 1981-2010. Sci Rep. 2014;4:6747. doi: 10.1038/srep06747.

3. Burris HA III, Moore MJ, Anderson J, et al. Improvements in survival and clinical benefits with gemcitabine as first-line therapy for patients with advanced pancreas cancer: a randomized trial. J Clin Oncol 1997;15:2403-13.

4. Le C, Sridhara R, Mahjoob K. Statistical Review and Evaluation of Erlotinib (OSI-774, Tarceva); FDA Statistical Review/NDA21-743/SE1-003. May 2005.

5. Moore MJ, Goldstein D, Hamm J, et al. Erlotinib plus gemcitabine compared with gemcitabine alone in patients with advanced pancreatic cancer: a phase III trial of the National Cancer Institute of Canada Clinical Trials Group. J Clin Oncol 2007;25:1960-6.

6. Conroy T, Desseigne F, Ychou M, et al. FOLFIRINOX versus Gemcitabine for Metastatic Pancreatic Cancer. N Engl J Med. 2011 May 12; 364 (19):1817-25. doi: 10.1056/NEJMoa1011923.
7. Von Hoff D, Ervin T, Arena F, et al. Increased Survival in Pancreatic Cancer with nab-Paclitaxel plus Gemcitabine. N Engl J Med. 2013 Oct 31; 369(18):1691-703. doi: 10.1056/NEJMoa1304369. Epub 2013 Oct 16.

8. [Internet] National Comprehensive Cancer Network. NCCN Clinical Practice Guideline in Oncology, Pancreatic Adenocarcinoma; 2016; Version 1. http://www.nccn.org/professionals/physician_gls/pdf/pancreatic.pdf. Accessed 2/1/2017

9. Qi F, Zhao L, Zhou A et. al. The advantages of using traditional Chinese medicine as an adjunctive therapy in the whole course of cancer treatment instead of only terminal stage of cancer. Biosci Trends 2015; 9(1): 16-34

10. Liu Y, Zhang W, Wang XJ, Liu S1. Antitumor effect of Kanglaite ${ }^{\circledR}$ injection in human pancreatic cancer xenografts. BMC Complement Altern Med. 2014 Jul 8;14:228. doi: 10.1186/1472-6882-14-228.

11. Lu Y1, Wu LQ, Dong O, Li CS. Experimental study on the effect of Kang-Lai-Te induced apoptosis of human hepatoma carcinoma cell HepG2. Hepatobiliary Pancreat Dis Int. 2009 Jun;8(3):267-72.

12. Woo JH1, Li D, Wilsbach K, Orita H, Coulter J, Tully E, Kwon TK, Xu S, Gabrielson E. Coix seed extract, a commonly used treatment for cancer in China, inhibits NFkappaB and protein kinase C signaling. Cancer Biol Ther. 2007 Dec;6(12):2005-11. Epub 2007 Oct 13.

13. Wheeler RH, Busby L, Samlowski W, Gerard R, Farling H. Phase I study of kanglaite (KLT) a botanical product based on traditional Chinese medicine. Proc Am Soc Clin Oncol. 2003;22:Abstract990.

14. Sun Y, Li Y, Qin S et al. A multicenter randomized phase II trial on Kanglaite Injection (KLT) plus gemcitabine hydrochloride (GEM) versus GEM in patients with local advanced and metastatic pancreatic cancer. Journal Clin Onc 2011; 29: Abstract-e14510

15. Bao Y, Xia L, Jang H. The experiment and study on cellular apoptosis produced by KLT injections in pancreatic cancer cells. Shanghai Journal Medicine 2004; 27: 421-424.

16. Li Y, Shi TZ. Mechanisms of Kanglaite induced apoptosis in human cancer cells. Chinese Journal of Clinical Oncology 2002; 29:869-872.

17. Yuan YZ, Bao Y, Xia L. The study on KLT-induced apoptosis of human pancreatic cancer Paru- 8988 cell which detected by Gene chip. Chinese Journal of Digestion 2004; 24:451-454.

18. Han SX, Zhu Q, Du BR. The mechanism of coixenolide-induced apoptosis in human cervical cancer cells. Oncology 2002; 22:481-482.

19. Huang T, Lv G, Gao DX,Wang YF. Experimental study on apoptosis of osteosarcoma cells induced by Kang-Lai-Te combined with doxorubicin. Chinese Journal of Histochemistry and Cytochemistry 2005; 14:648-652.

20. Wu LQ, Lu Y, Lu HJ. Efficacy of intra-tumor injection of Kang-Lai-Te in treating transplanted hepatoma in rats. Hepatobiliary \& Pancreatic Diseases International 2004; 3:580-584

21. Liu Y, Zhang W, Wang X-J, Liu S. Antitumor effect of Kanglaite injection in human pancreatic cancer xenografts. BMC complementary and Alternative Medicine 2014:28

22. Liu X, Xu F, Wang G, Dino X, Li Y. Kanglaite injection plus chemotherapy vs. chemotherapy alone for non-small cell lung cancer patients: A systematic review and meta-analysis. Current Therapeutic Research 2008; 69:381-411.

23. Zhan Y-P, Huang X-E, Cao J, Lu Y-Y, Wu X-Y, Liu J, Xu X, Xiang J, Ye L-H. Clinical safety and efficacy of Kanglaite (coix seed oil) injection combined with chemotherapy in treating patients with gastric cancer. Asian Pacific J. Cancer Prevention 2012; 13(10): 5319-5321.

24. Qi F, Li A, Inagaki Y, Gao J, Li J, Kokudo N, Li YK, Tang W. Chinese herbal medicines as adjuvant treatment during chemo- or radio-therapy for cancer. Bioscience Trends 2010; 4(6): 297-307. 